Archives

40 | 2007

Dépendance(s)

\title{
De l'accomplissement de soi à la dépendance psychologique
}

De quelques produits paradoxaux de l'individualisme spirituel contemporain *

Danièle Hervieu-Léger

\section{(2) OpenEdition}

\section{Journals}

Édition électronique

URL : http://journals.openedition.org/ccrh/3341

DOI : $10.4000 /$ ccrh.3341

ISSN : $1760-7906$

Éditeur

Centre de recherches historiques - EHESS

\section{Édition imprimée}

Date de publication : 25 avril 2007

Pagination : 67-76

ISSN : 0990-9141

\section{Référence électronique}

Danièle Hervieu-Léger, « De l'accomplissement de soi à la dépendance psychologique », Les Cahiers du Centre de Recherches Historiques [En ligne], 40 | 2007, mis en ligne le 11 octobre 2011, consulté le 02 mai 2019. URL : http://journals.openedition.org/ccrh/3341 ; DOI : 10.4000/ccrh.3341

Ce document a été généré automatiquement le 2 mai 2019.

Article L.111-1 du Code de la propriété intellectuelle. 


\section{De l'accomplissement de soi à la dépendance psychologique}

De quelques produits paradoxaux de l'individualisme spirituel contemporain $*_{1}$

Danièle Hervieu-Léger

\section{Les régimes contemporains de la validation du croire}

1 Le paysage religieux de la modernité est caractérisé par un mouvement irrésistible d'individualisation et de subjectivisation des croyances et des pratiques. Cette proposition - déclinée à l'infini à partir des observations conduites dans toutes les sociétés occidentales - constitue aujourd'hui le leitmotiv de la réflexion sociologique sur les formes contemporaines du religieux. Mais la formule soulève immédiatement une question : celle des formes de sociabilité religieuse qui sont encore susceptibles d'exister lorsque s'impose, de façon aussi massive, un individualisme religieux pleinement intégré à l'individualisme moderne.

2 Si l'individu produit lui-même, de façon autonome, le petit « dispositif du sens » qui lui permet d'orienter sa propre vie et de répondre aux «questions ultimes» de son existence, si son expérience spirituelle se condense dans une relation intime et purement privée avec ce qu'il choisit ou non de nommer Dieu, si cette expérience éminemment personnelle ne prescrit pas une action dans le monde, l'appartenance à une communauté croyante devient alors secondaire, sinon complètement inutile. On peut même imaginer que la logique contem-poraine du «bricolage croyant » rende impossible la constitution de commu-nautés croyantes réunies par une foi partagée. Dans cette hypothèse limite, l'actualisation communautaire d'une lignée croyante quelconque - cette référence à la continuité d'une tradition qui fait la substance même du lien religieux - tend à disparaître. L'expansion de la spiritualité moderne de l'individu pourrait bien signer, de ce point de vue, la fin de la religion. Car l'atomisation des quêtes spirituelles individuelles ne décompose pas seulement le lien religieux constitué dans l'attestation d'une vérité partagée par une communauté passée, présente et future : elle interdit en même temps, 
au nom d'une conception purement subjective de la vérité à atteindre, la recomposition de ce lien, sous quelque forme que ce soit. Le régime purement subjectif de la vérité auquel conduit l'atomisation individualiste des croyances peut préserver une forme de religiosité individuelle (le fait, pour un individu, de reconnaitre son affinité subjective avec telle ou telle tradition croyante), mais il dissout, potentiellement, toute forme de communalisation religieuse.

3 Ce schéma constitue évidemment une tendance limite. Il ne se réalise concrètement que lorsque s'impose un régime d'autovalidation du croire, dans lequel le sujet croyant ne reconnaît qu'à lui-même la capacité de définir la vérité du croire. Cette tendance existe dans certains courants spirituels contemporains, mais elle est loin d'être exclusive. La dissémination du croire suscite également un mouvement tout contraire de prolifération communautaire. Fait que l'on peut résumer de la façon suivante: plus les individus «bricolent » le petit système de croire ajusté à leurs besoins propres, et plus ils aspirent à exprimer et à échanger cette expérience avec d'autres individus qui partagent le même type d'aspirations spirituelles. Cette contradiction apparente correspond en fait aux limites intrinsèques de l'autovalidation du croire. Pour que les individus parviennent à stabiliser les significations qu'ils produisent afin de donner un sens à leur expérience quotidienne, ils ont besoin de trouver en dehors d'eux une confirmation de leur validité. Privés des confirmations fortes qu'offraient les codes globaux du sens garantis par les institutions (systèmes religieux ou philosophiques, idéologies politiques etc.), c'est avant tout dans l'échange mutuel que les individus peuvent espérer trouver les moyens de consolider l'univers personnel de sens dont ils se dotent. Dans ce cas, l'autovalidation peut laisser la place à un régime de validation mutuelle $d u$ croire, fondé sur le témoignage personnel, l'échange des expériences individuelles, et éventuellement sur la recherche des voies de leur approfondissement collectif.

4 Ce régime de la validation mutuelle n'est pas seulement au principe de la constitution des réseaux mouvants de la nébuleuse mystique-ésotérique. Il envahit également le monde des religions instituées. Le paysage actuel des Églises est caractérisé par le développement de groupes et réseaux qui mettent en œuvre, en marge ou au cœur des paroisses et des mouvements, des formes souples et mouvantes de sociabilité affinitaire, fondées sur la proximité spirituelle, sociale et culturelle des individus qui y sont impliqués.

5 Le développement de ces formes mobiles, poreuses et souvent instables de sociabilité religieuse correspondant au régime de la validation mutuelle du croire cohabite par ailleurs avec une autre tendance, apparemment toute contraire, à la montée en puissance des formes de communalisation intensive qui se déploient, à l'intérieur et à l'extérieur des grandes traditions religieuses, à l'intérieur et à l'extérieur des grandes traditions religieuses, à partir d'un régime de validation communautaire du croire. Dans ce dernier cas, des croyants convaincus investissent des certitudes partagées dans des formes communes d'organisation de la vie quotidienne et/ou d'action dans le monde. Le modèle militant du « mouvement » - mais également le modèle « monastique » d'une vie religieuse intégrale vécue en dehors du monde - impliquent, entre ceux qui se rassemblent selon l'un de ces modes, l'adhésion à un régime commun du croire gagé, si l'on peut dire, sur l'intensité de leur engagement individuel et collectif. La cohésion communautaire atteste, pour chacun, la vérité du croire commun.

6 Les religions instituées font prévaloir, en principe, un régime institutionnel de la validation $d u$ croire, mis en œuvre par des instances garantes, selon le type d'organisation du 
pouvoir propre à chaque tradition, de la continuité de la, lignée croyante. Celles-ci définissent des règles d'orthodoxie et d'orthopraxie qui sont, pour les individus, les repères stables de la conformité croyante. Ce régime qui vaut pour l'ensemble des fidèles n'exclut pas que se différencient, en son sein, des régimes de validation communautaire $\mathrm{du}$ croire répondant au désir d'intensité religieuse de groupes particuliers. Des communautés unissent ainsi de façon plus étroite et plus spécifique des familles spirituelles qui partagent une interprétation commune du rapport au monde et du mode de vie qu'implique la possession de cette vérité commune. Les congrégations et ordres religieux, les mouvements, les groupes de dévotion, les communautés dites "nouvelles » inscrivent, non sans de fréquents conflits dont l'histoire des fondations d'ordres religieux porte particulièrement la trace, leur propre régime de validation à l'intérieur du régime général de la validation institutionnelle. Mais cette validation communautaire du croire peut également se développer de façon autonome, en opposant au «plus petit dénominateur croyant » que les institutions tendent à instaurer entre leurs fidèles, un régime intensif de la vérité partagée entre des individus personnellement convertis. Celui-ci implique une démarche personnelle d'adhésion de la part des individus regroupés dans la communauté, et c'est l'intensité de l'engagement pris par chacun qui valide, pour les autres, les significations partagées au sein du groupe. La montée de l'individualisme religieux a renforcé, face aux définitions institutionnelles du croire formellement partagées par un peuple de fidèles, l'affirmation plurielle de régimes communautaires du croire, liant contractuellement des individus également impliqués dans leur vie religieuse.

\section{Un régime transverse : celui de la validation charismatique du croire}

7 À ces différents régimes typiques de la validation du croire, qui peuvent éventuellement se combiner, il faut ajouter un autre dispositif de validation: celui qui passe par l'intervention et le témoignage de personnalités exceptionnelles que leur expérience qualifie pour servir de guides à ceux qui se reconnaissent en elles. Le porteur de charisme trouve, dans la «communauté émotionnelle» des disciples qui l'entourent, la confirmation de sa propre élection charismatique, mais ce jeu de la reconnaissance réciproque peut s'inscrire aussi bien dans les règles de la validation communautaire $d u$ croire que dans celles de la validation mutuelle du croire. Le porteur de charisme peut définir des normes communautaires offertes à l'adhésion de chacun des adeptes. Il peut simplement ouvrir un chemin aux individus qui identifient leur expérience à la sienne. Il peut même, dans le cas où il s'agit d'un charisme de fonction, s'inscrire en le renforçant dans un dispositif de validation institutionnelle du croire. La validation charismatique du croire ne constitue pas, comme telle, un régime spécifique de validation du croire, donnant lieu à une forme particulière de communalisation religieuse : elle traverse en fait les régimes précédemment identifiés, en assurant notamment le passage de l'un à l'autre. L'extrême individualisation du croire qui caractérise le paysage religieux de la modernité avancée, l'affaissement des régimes institutionnels de la validation du croire et le besoin montant de confirmation mutuelle et communautaire des "petites vérités " produites par les individus favorise considérablement le rôle de personnalités qui peuvent témoigner, par leur expérience personnelle, d'une antériorité sur le chemin de la vérité et donc d'une capacité d'initier ceux qui sont disposés à les suivre. De Desmond 
Tutu à l'Abbé Pierre, en passant par Mère Theresa, le Dalaï Lama et Jean-Paul II, des figures religieuses ont exercé et exercent une fascination sur l'opinion qui contraste singulièrement, notamment chez les jeunes, avec la faible confiance accordée par ailleurs aux institutions religieuses. L'aura qui entoure ces «héros spirituels» n'est pas sans rapport avec la spectacularisation généralisée de la vie sociale qui correspond à l'avènement de la civilisation des médias. Leur succès entretient également un lien certain avec une culture de la performance qui valorise tous ceux, quel que soit le domaine dans lequel ils déploient leurs activités, qui sont parvenus à sortir des sentiers battus, à s'arracher aux routines de la vie ordinaire et à «aller jusqu'au bout d'euxmêmes ».

8 La perte de plausibilité des systèmes de sens formatés par les institutions tend à conférer à des grands témoins constitués en hommes-sens (ou en femmes-sens) une "capacité validatrice» croissante. Ces figures charismatiques - prophètes, sages, gourous, bergers etc. - qui relaient à différents niveaux les figures traditionnelles du pouvoir religieux prêtres, pasteurs, anciens et autres chefs de communautés - constituent en même temps des maillons mobiles, qui assurent couramment le passage des individus d'un mode de validation du croire à un autre, et ceci dans tous les sens. Dans la perspective weberienne classique, qui fait du charisme « la force de changement des époques liées à la tradition ", le « porteur d'un charisme prophétique » se distingue et s'affirme en se prévalant d'une « révélation personnelle » et neuve, qui inaugure, pour ceux qui la reçoivent, un nouveau mode de vie. Le succès des "personnalités charismatiques" tient, avant tout, dans les sociétés gouvernées par la culture de l'individu, à ce que celles-ci font valoir une expérience vécue originale. L'intensité et la qualité de leur engagement personnel définit exactement leur capacité normative vis-à-vis d'autrui et l'étendue de la reconnaissance à laquelle elles peuvent prétendre. Cet engagement personnel entre en consonance avec la problématique moderne de la responsabilité autonome de l'individu. Il fait d'elles les porteurs privilégiés du régime de la validation mutuelle du croire.

Mais du fait même de l'autorité que ces « individus accomplis » constituent ainsi au titre de leur "aînesse spirituelle ", ils peuvent cristalliser autour d'eux - et ceci au-dehors aussi bien qu'au-dedans des grandes églises - des communautés de disciples au sein desquelles s'impose souvent, au moins au bout d'un certain temps, un régime communautaire de la validation du croire. Ils peuvent ainsi contribuer, par la seule vertu de leur aura personnelle, à faire évoluer un réseau spirituel affinitaire fonctionnant en régime de validation mutuelle, vers un régime plus structuré de la validation communautaire du croire partagé, dont ils se posent alors en garants. La dynamique d'un certain nombre de communautés nouvelles au sein desquelles la personnalité du fondateur a progressivement concentré l'adhésion de membres réunis initialement par des affinités spirituelles partagées entre eux de façon égalitaire, voire relativement lâche, illustre bien ce mouvement. Enfin, lorsqu'ils peuvent se prévaloir à la fois d'une autorité institutionnelle formelle et d'une capacité charismatique personnelle, ces leaders peuvent jouer un rôle dans la "mise en conformité » institutionnelle de groupes ou de réseaux organisés d'abord plutôt sur la base d'une validation communautaire ou mutuelle du croire commun. Un certain nombre de prêtres, de pasteurs ou de rabbins, reçus comme des "personnalités prophétiques » au sein de groupes aspirant à bénéficier de la communication de leur expérience, jouent clairement ce rôle de médiateurs institutionnels : leur charisme personnel, en rechargeant l'efficacité de leur « charisme de fonction ", permet, dans un certain nombre de cas, de restaurer un régime de validation 
institutionnelle du croire, par-delà la dissémination des petits récits croyants, communautairement ou mutuellement validés.

\section{Les « groupements de pratiquants »}

10 Cette dynamique est particulièrement lisible au sein des groupements de pratiquants, dans lesquels est proposée et mise en œuvre une méthode plus ou moins systématisée d'accès à des bénéfices spirituels partagés: activités rituelles, prière, exercices de méditation, enseignement et étude, prescriptions éthiques et «hygiène spirituelle» de vie etc. On pourrait aligner ici de nombreux exemples, pris dans toutes les traditions. On en retiendra un seulement, pour illustrer le propos, celui de la Soka Gakkaï, secte bouddhiste japonaise qui a connu une forte expansion partout dans le monde depuis trente ans. Celle-ci propose aux adeptes larécitation la plus fréquente possible d'un mantra devant le parchemin sacré, ou Gohonzon.L'individu qui organise sa vie autour de cette pratique est supposé entrer progressivement dans la connaissance de la loi qui régit tous les aspects de la vie. Il transforme son rapport à lui-même et aux autres, il augmente ses possibilités de réalisation personnelle dans le monde et entre dans une «troisième civilisation » qui dépasse le christianisme et la civilisation matérielle aujourd'hui dominante, et ouvre sur un monde de paix. Les implications poli-tiques contestées de cette dernière visée nous intéressent moins ici que le lien directement - et même mécaniquement - établi entre une pratique particulière minutieusement codée et le changement intérieur. La pratique, avant d'être l'extériorisation de la foi de l'individu, est considérée comme le moyen directement opératoire d'une transformation personnelle, dont les résultats concrets sont présentés comme assurés. Cet exemple, pris parmi une multitude d'autres possibles, illustre bien la montée en puissance d'une conception pragmatique et instrumentale de la pratique, mise en œuvre comme moyen d'accès à des bienfaits concrets en ce monde : une problématique qui diffuse, de façon plus ou moins explicite dans l'ensemble, des courants spirituels qui entretiennent un lien direct avec la culture moderne de l'efficacité.

11 On comprend que la notion de "pratique " n'a pas ici le sens restreint qu'on lui donne couramment lorsqu'on identifie la "pratique religieuse » à l'observance cultuelle régulière - définie par l'assistance à la messe du dimanche - selon le modèle catholique romain. Elle s'étend même au-delà de l'acception plus générale qu'on lui donne en définissant le "pratiquant» ou l'«observant» comme celui qui conforme son comportement aux pratiques qu'une institution religieuse particulière associe, de façon obligatoire, aux croyances qu'elle soutient. On parle, dans ce sens, des «juifs pratiquants » qui mettent en œuvre de façon scrupuleuse les différents mistvot, ou des « musulmans pratiquants » qui respectent fidèlement les piliers de l'islam. Cette figure du " pratiquant comme observant » a incarné longtemps (et continue d'incarner, du point de vue des institutions religieuses) un idéal de fidélité religieuse, par rapport auquel il est possible de décliner - du plus conforme au moins conforme - des modalités inégalement «régulières» de la pratique. Dans cette perspective, c'est la norme institutionnelle qui définit le "pratiquant». Or, cette approche se trouve aujourd'hui mise en question: le pratiquant n'est plus (ou de moins en moins) un individu qui se plie par obligation à des normes religieuses fixées en dehors de lui. Il revendique, beaucoup plus couramment, d'assumer comme un choix les implications pratiques correspondant à son engagement croyant, en vue de l'accomplissement personnel auquel il prétend. 
12 Ce changement de sens de la pratique peut opérer même lorsque les comportements individuels continuent de s'aligner, de fait, sur des normes institutionnelles présentées comme des obligations : ainsi, il est de plus en plus rare, surtout dans les générations les plus jeunes, qu'un individu qui se rend à la messe tous les dimanches justifie sa propre régularité observante en invoquant d'emblée «la loi de l'Église». Il soulignera plus volontiers l'«équilibre personnel» qu'il a trouvé dans le «rythme religieux» (la « respiration») que la fréquentation hebdomadaire de l'Église donne à son existence quotidienne, ou bien il fera valoir le «besoin intérieur " qu'il ressent à participer au rassemblement communautaire dominical. Le sens de la pratique s'établit, autrement dit, à partir de l'individu.

13 Ceci est vrai, a fortiori, lorsque l'engagement croyant met d'emblée l'accent sur l'implication personnelle du sujet dans une activité spirituelle ou religieuse dont le but explicite est l'accès à la réalisation plénière de soi-même. Ainsi, les « convertis » français au bouddhisme qui s'engagent dans une pratique régulière de la méditation disent couramment le faire en vue d'une augmentation de leur potentiel spirituel personnel. L'acceptation des règles qui régissent la méditation trouve son entière justification dans l'efficacité que ces principes assurent à la quête spirituelle personnelle. De façon générale, la "pratique » tend donc à se définir comme une discipline volontairement acceptée pour accéder aux biens spirituels recherchés, et elle embrasse à ce titre tous les actes et comportements que l'individu associe expressément à l'obtention de ces biens.

\section{De la discipline à la dépendance : le choc en retour du prix à payer pour l'accès à la réalisation de soi}

14 La plupart du temps, la mise en œuvre de cette discipline nécessite une préparation et une formation spécifique sous la conduite d'un spécialiste ; ou bien elle appelle l'initiation d'un maître ou encore le contrôle d'un guide spirituel ou d'un berger témoignant personnellement du chemin offert au candidat à l'accomplissement.

Si ce chemin peut parfois être proposé comme une voie purement individuelle que chacun parcourt à son rythme, il requiert couramment le soutien d'un groupe au sein duquel les trajectoires individuelles sont validées et évaluées. Les groupements de pratiquants assurent, sous une forme ou sous une autre, la socialisation qui permet que la pratique se construise comme activité permanente, définissant et structurant l'identité spirituelle/religieuse de ceux qui s'y livrent ensemble. L'organisation de ces groupements de pratiquants varie considérablement selon la nature de l'exercice spirituel proposé aux membres, selon l'intensité de l'engagement que cet exercice requiert d'eux, et selon le type de pouvoir qui en assure la direction et le contrôle. On pourrait consacrer des livres entiers à la description empirique des modes d'organisation et des formes de sociabilité repérables au sein de la population des groupements de pratiquants. Bornons-nous simplement à remarquer qu'ils se distribuent entre un "pôle faible", défini par des conditions minimales de participation compatibles avec les engagements de la vie ordinaire dans le monde, et un " pôle fort » où la pratique tend - ultimement - à englober et à ordonner tous les domaines de la vie. Plutôt qu'à l'inventaire des formes et degré de la pratique qui s'établissent entre ces deux pôles, il faut s'attacher à souligner l'alignement tendanciel des conceptions de l'activité spirituelle et religieuse (aussi bien individuelle que collective) sur des problématiques de l'exercice et de l'entraînement 
(training) qui participent d'une culture de l'efficacité pragmatique devenue la norme dans tous les domaines de la vie sociale.

On fera valoir que la notion d'«exercice spirituel " n'a rien, en tant que telle, de véritablement neuf, et que toutes les traditions religieuses ascétiques et mystiques ont mis au point depuis très longtemps des disciplines pratiques destinées à ouvrir à ceux qui s'y soumettent une voie de perfection ou d'illumination (ou au moins à leur en faciliter l'accès). La spécificité des pratiques contemporaines de l'« entraînement spirituel » tient à l'affinité qu'elles entre-tiennent avec une culture de l'épanouissement de soi dont l'idéal moral (celui qui définit ce que serait une existence meilleure ou plus élevée) est, ultimement, celui de la présence authentique à soi-même. La " pratique " ainsi entendue garde la fonction d'introduire l'individu dans un autre ordre de réalité, de l'inscrire dans un cosmos sacré, ou de lui assigner une place dans un dispositif institué de médiation assurant de façon exclusive la communication avec la transcendance. Mais sa fonction première est, avant tout, de le ramener à lui-même et à sa vie ordinaire en lui permettant de maximiser ses propres potentialités, d'accéder à la paix intérieure, de trouver l'ordre intime de sa propre vie et, éventuellement, d'augmenter ses performances dans le monde.

Entendons bien que cette imprégnation de la pratique spirituelle et religieuse par l'individualisme expressif ne fait pas disparaitre toutes les autres conceptions du sens de l'activité religieuse. Cette conception de la «pratique » n'absorbe pas entièrement les définitions de la fidélité religieuse qui se sont élaborées au sein des grandes institutions religieuses, mais elle pousse à la limite une dynamique de l'intériorisation spirituelle qui constitue, au moins depuis la Réforme, la trame même de la modernisation de la religion. Elle ne concerne pas, à ce titre, les seules religions ou spiritualités émergentes qui thématisent directement l'objectif de la réalisation de soi comme finalité de l'activité spirituelle. Elle « travaille» les groupements religieux traditionnels et les « communautés nouvelles » qui prolifèrent au sein des grandes églises. Elle constitue évidemment l'un des supports de l'expansion de certains courants religieux - de l'évangélisme protestant au charismatisme catholique - dont la dynamique conversionniste et émotionnelle favorise particulièrement la reformulation de la conception chrétienne du salut et de la Grâce à l'intérieur de l'univers culturel de l'individualisme expressif.

On ne manquera pas de remarquer que le choix personnel d'entrer dans une "discipline " spirituelle rationalisée au sein d'un groupe, sous la direction d'un guide ou d'un maitre, peut conduire des individus - qui valorisent pourtant à l'extrême la " voie personnelle » qu'ils entendent explorer - à accepter un encadrement normatif extrêmement poussé de leur vie quotidienne. C'est le cas, par exemple, d'un certain nombre de convertis au bouddhisme d'origine catholique, qui sont particulièrement nombreux en France : parmi les premières raisons que les intéressés avancent pour justifier leur conversion, il y a la contrainte normative qu'ils disent avoir vécu au sein de l'Église. Il n'est pas rare d'ailleurs que certains livrent des tableaux de la «tyrannie cléricale» ou du "sadisme des religieuses » dont on doute quelque peu, compte tenu de leur âge et de leur parcours, qu'ils en aient jamais eu l'expérience directe... Mais le cliché l'emporte, et permet de valoriser, a contrario, l'ouverture tolérante de la nouvelle voie qu'ils ont choisie, le respect accordé à la spécificité de leur recherche personnelle et l'absence de prescriptions morales et rituelles rigides qui caractérise, selon eux, le bouddhisme. Or, dans la pratique de cette nouvelle voie, les mêmes se placent couramment dans une dépendance psychologique extrême à l'égard du maître qu'ils se sont donnés, attendant tout de ses avis et prêts à se ranger à toutes ses prescriptions, si celui-ci accepte - ce qui n'est pas 
exceptionnel - d'entrer dans le jeu de cette dépendance. Il y a là en effet un paradoxe, mais qui renvoie, plus fondamentalement, aux contradictions de la demande spirituelle elle-même, prise entre l'affirmation des droits absolus de la subjectivité (le droit de chacun à trouver sa voie d'accomplissement), et le besoin de mise en ordre de la vie personnelle qui requiert des repères suffisamment fixes.

Ces repères peuvent être trouvés dans les règles d'observance parfois très contraignantes que l'individu accepte avec le sentiment fort de se les être données à lui-même. Ils procèdent, de façon extrêmement fréquente, de l'attachement affectif à un individu doté d'un fort charisme personnel dont l'expérience exceptionnelle fonde la capacité de fixer des normes pour autrui. Dans tous les cas, le fait d'« avoir choisi » le cadre d'exercice de la pratique et de s'être "donné à soi-même" les objectifs spirituels qui justifient d'accepter les moyens permettant d'y accéder permet, à tout moment, de transformer la soumission en une adhésion volontaire qui fait partie intégrante de la recherche d'accomplissement personnel poursuivie par l'individu. Il est indispensable de saisir ce ressort fondamentalement individualiste de l'adhésion pour éclairer les contradictions apparentes de la participation à des groupements de pratiquants qui apparaissent, vus de l'extérieur, comme des structures oppressives, exerçant une emprise totalisante sur la vie de leurs membres. La soumission requise des adeptes au sein de ces groupes y est couramment convertie - par les intéressés eux-mêmes - en une démonstration de virtuosité, cohérente avec (et nécessaire à) la performance spirituelle personnelle qu'ils visent.

Se noue ici le malentendu dramatique qui oppose couramment le pratiquant intensif ou virtuose à ses proches : là où ceux-ci ne constatent que la dépendance (une dépendance qu'ils imputent spontanément à une «manipulation»), l'intéressé fait valoir les impératifs, entièrement voulus par lui, d'un exercice ordonné à la réalisation d'une performance spirituelle personnelle. Il faut rappeler au passage que ce malentendu n'est en rien caractéristique des groupes désignés comme des sectes, mais qu'il accompagne, de façon à peu près inévitable, les conflits de la radicalité, confrontée aux standards sociaux d'une vie religieuse considérée comme "normale». Bien des parents, découvrant la vocation monastique de leur fils ou de leur fille et horrifiés à l'idée que celui ou celle-ci puissent «s'enfermer au couvent », ont éprouvé les impasses de l'argumentation portant sur le «renoncement à la liberté", face à des individus faisant valoir, dans ce renoncement même, l'exercice radical de leur liberté personnelle. Et un bon nombre d'entre eux n'ont sans doute pas manqué d'invoquer, en faisant l'expérience de ce dialogue impossible, l'influence manipulatrice d'un aumônier exerçant une pression abusive sur les consciences... La pluralisation du paysage religieux et spirituel contemporain et la diversification des groupements de pratiquants qui lui correspond a fait exploser ce conflit de la radicalité en-dehors des espaces de la virtuosité religieuse (les monastères, les couvents et ordres religieux, la vie sacerdotale elle-même etc.), placés sous le contrôle d'une institution religieuse reconnue et plus ou moins acclimatés socialement. Par ailleurs, l'expansion d'une culture de la performance spirituelle rapportée à la réalisation personnelle de soi-même l'emporte aujourd'hui largement sur la culture du dépouillement de soi qui a orienté, pendant des siècles, une virtuosité religieuse ordonnée avant tout à l'accès au salut dans un autre monde. Les formes contemporaines de la pratique virtuose - avant de constituer une « dérive » aberrante de la religiosité - se présentent donc (et doivent être traitées) comme des modalités ultraintensives d'une démarche d'individuation spirituelle, cohérente avec l'horizon moderne 
de l'accomplissement de soi : elles sont une des expressions d'une culture moderne de l'extrême qui se développe également sur d'autres terrains, celui de la performance sportive, du voyage, du raid ou de la traversée de l'océan, menés en solitaire. Ces expériences ont toutes en propre, comme l'expérience mystique, de faire jouer simultanément la recherche de l'autonomie absolue de l'individu (qui repousse toujours plus loin la limite) et l'acceptation de cette dépendance très particulière qui procède de la fascination même que cette limite exerce sur ceux qui se confrontent à elle.

\section{NOTES}

1. * NB : ce texte recoupe de façon exagérément ramassée, des réflexions dont on trouvera la présentation développée dans deux ouvrages: Danièle Hervieu-Léger, Le pèlerin et le converti. La religion en mouvement, Paris, Flammarion, 1999 (Coll. Champs, 2001) (trad. en espagnol, allemand, italien, portugais). Chapitre 5 ; id., La religion en miettes ou la question des sectes, Paris, CalmannLévy, 2001, chap. 5.

\section{AUTEUR}

DANIÈLE HERVIEU-LÉGER

EHESS/CEIFR 